\title{
Association of Tagging SNPs in the MTHFR Gene with Risk of Type 2 Diabetes Mellitus and Serum Homocysteine Levels in a Chinese Population
}

\author{
Han Wang, ${ }^{1}$ Cong Hu, ${ }^{2}$ Shu-Hui Xiao, ${ }^{3}$ and Bin Wan ${ }^{2}$ \\ ${ }^{1}$ Undergraduate Student Brigade 11 Battalion, Third Military Medical University, Chongqing 400038, China \\ ${ }^{2}$ Center for Reproductive Medicine, The First Affiliated Hospital of Jilin University, Changchun 130062, China \\ ${ }^{3}$ Laboratory of Immune, People’s Hospital of Linyi, No. 27 Eastern Jiefang Road, Lanshan, Linyi 276003, China \\ Correspondence should be addressed to Shu-Hui Xiao; xiaoshuhui@team301.org
}

Received 17 June 2014; Revised 22 July 2014; Accepted 24 July 2014; Published 6 August 2014

Academic Editor: Seul-Ki Jeong

Copyright (C) 2014 Han Wang et al. This is an open access article distributed under the Creative Commons Attribution License, which permits unrestricted use, distribution, and reproduction in any medium, provided the original work is properly cited.

\begin{abstract}
Diabetes is a global public health crisis, and the prevalence is increasing rapidly. Folate supplementation is proved to be effective in reducing the risk of diabetes or improving its symptoms. Methylenetetrahydrofolate reductase is an important enzyme involved in folate metabolism. The aim of this study is to examine whether polymorphisms in the MTHFR gene are associated with risk of type 2 diabetes mellitus (T2DM) and fasting total serum homocysteine (tHcy) levels. We genotyped nine tagging SNPs in the MTHFR gene in a case-control study, including 595 T2DM cases and 681 healthy controls in China. We found that C allele of rs9651118 had significant decreased risk of T2DM (adjusted odds ratio $(\mathrm{OR})=0.69,95 \%$ confidence interval $(\mathrm{CI}): 0.55-0.87, P=$ 0.002) compared with T allele. Haplotype analysis also showed that MTHFR CTCCGA haplotype (rs12121543-rs13306553-rs9651118rs1801133-rs2274976-rs1801131) had significant reduced risk of T2DM (adjusted OR $=0.71,95 \%$ CI: $0.58-0.87, P=0.001$ ) compared with CTTTGA haplotype. Besides, the MTHFR rs1801133 was significantly associated with serum levels of tHcy in healthy controls $(P=0.0002)$. These associations were still significant after Bonferroni corrections $(P<0.0056)$. These findings suggest that variants in the MTHFR gene may influence the risk of T2DM and tHcy levels.
\end{abstract}

\section{Introduction}

Diabetes mellitus (DM) is one of the primary chronic diseases that causes premature death and disability. It was estimated to be the third most challenging disease that is threatening public health just after malignant tumors and cardiocerebral vascular diseases in the world [1]. In recent years, the prevalence of DM was rising rapidly worldwide, particularly in developing countries [2]. In China, age-standardized incidence rate of DM was $2.4 \%$ in 1994 but raised to $9.7 \%$ in 2007-2008 [3, 4]. The importance of conducting prevention and early detection of DM has been increasingly emphasized.

Type $2 \mathrm{DM}$ (T2DM) is the most common form of diabetes, which accounts for about $90 \%$ of all diagnosed DM cases. It is a multifactorial disease that is due to a combination of environmental and genetic risk factors, including dietary habit, obesity, physical inactivity, smoking, alcohol consumption, and genetic variants [5]. Studies have confirmed the relationship between homocysteinemia and diabetic complications, such as diabetic nephropathy, cardiovascular disease, and hypertension [6-8]. It is also reported that a high concentration of homocysteine is associated with the development of T2DM $[9,10]$. MTHFR (methylenetetrahydrofolate reductase), a folate-dependent enzyme, plays an important role in the conversion of homocysteine to methionine by converting 5,10-methylenetetrahydrofolate to 5-methyltetrahydrofolate. Studies have found that specific genetic polymorphisms in the MTHFR gene could lead to change of MTHFR enzyme activity [11, 12]. Previous epidemiologic studies have also shown that genetic polymorphisms in MTHFR gene may 
be related to cancers, ischemic stroke, hypertension, and so forth [13-15]. But the association of MTHFR genotypes and susceptibility to T2DM in Chinese population has not been fully studied.

To help clarify whether the MTHFR variants are associated with susceptibility of T2DM and serum total homocysteine (tHcy) level, we examined nine tagging SNPs in the MTHFR gene (rs12121543, rs13306561, rs13306553, rs9651118, rs1801133, rs2274976, rs4846048, rs1801131, and rs17037396) in a case-control study in China.

\section{Material and Methods}

2.1. Study Participants. In this study, we consecutively enrolled 595 T2DM patients in the first affiliated hospital of Jilin university, Changchun, China, aged from 35 to 80, between June 2011 and June 2013. A case of T2DM was considered to be confirmed if the participant's reported glucose level met the well-established diagnostic criteria recommended by American diabetes association: with either the fasting plasma glucose $(\mathrm{FPG})$ of $\geq 7.0 \mathrm{mmol} / \mathrm{L}$ or the 2 -h value in the 75 -g oral glucose tolerance test $(\mathrm{OGTT}) \geq 11.1 \mathrm{mmol} / \mathrm{L}$ [16]. A total of 655 healthy control subjects were selected during the same period and from the same hospital and were frequency matched to the cases by age (5-year age groups) and gender. All controls were individuals free of diabetes that are determined by medical history or clinical examinations. At enrollment, demographic and medical histories, including age, gender, body mass index, smoking habits, and hypertension status were collected from each subject by a trained interviewer using a structured questionnaire. Blood samples were collected after a 12-hour overnight fast and then separated into serum, red blood cells, and buffy coat. Written informed consent was obtained from all enrolled participants and this study was approved by the Ethics Committee of Jilin University.

2.2. SNP Selection. Tag SNPs were selected by searching Han Chinese data from the HapMap project (http://www.hapmap .org/) using the Tagger program. The following criteria were used to identify tagging SNPs: (a) SNPs located in the gene or within the $2-\mathrm{kb}$ region flanking the gene, (b) a minor allele frequency $\geq 0.1$, and (c) other unselected SNPs could be captured by one of the tagging SNPs with a linkage disequilibrium of $r^{2} \geq 0.90$. As a result, a total of 9 tag SNPs were identified.

2.3. Laboratory Tests. Serum levels of total cholesterol (TC), low density lipoprotein-cholesterol (LDL-C), and high density lipoprotein-cholesterol (HDL-C) were determined in cases and controls by commercial kits from the Nanjing Jiancheng Bio-company (Nanjing, China). Serum tHcy levels in healthy controls were measured by cycling enzymatic method (Nanjing Jiancheng Bio-company, Nanjing, China) and were analyzed with an automatic biochemical analyzer (AU 2700 Olympus, First Chemical Ltd, Tokyo, Japan).
TABLE 1: Selected characteristics of cases and controls.

\begin{tabular}{lccc}
\hline Characteristics & Cases $(n=595)$ & Controls $(n=681)$ & $P$ value \\
\hline Age (year) & $65(56-71)$ & $64(56-73)$ & 0.43 \\
Sex $($ male/female $)$ & $321 / 274$ & $375 / 306$ & 0.69 \\
BMI $\left(\mathrm{kg} / \mathrm{m}^{2}\right)$ & $24.6(22.7-27.0)$ & $23.1(21.1-25.4)$ & $<0.001$ \\
Smoking $($ yes/no) & $182 / 413$ & $229 / 452$ & 0.25 \\
$\begin{array}{l}\text { Hypertension } \\
\text { (yes/no })\end{array}$ & $261 / 334$ & $167 / 514$ & $<0.001$ \\
$\begin{array}{l}\text { Total cholesterol } \\
\text { (mmol/L) }\end{array}$ & $4.68(4.09-5.48)$ & $4.14(3.60-5.07)$ & $<0.001$ \\
HDL-C $(\mathrm{mmol} / \mathrm{L})$ & $1.10(0.98-1.42)$ & $1.24(1.09-1.58)$ & $<0.001$ \\
LDL-C $(\mathrm{mmol} / \mathrm{L})$ & $2.81(2.45-3.29)$ & $2.38(2.05-2.93)$ & $<0.001$ \\
\hline
\end{tabular}

Genomic DNA was extracted from peripheral blood leucocytes using DNA Extraction Kit (Qiagen, Hilden, Germany). Genotyping was performed on the ABI PRISM 7900HT Sequence Detection System (Applied Biosystems, Foster City, CA, USA), using the TaqMan assay. The genotyping call rate was $>95 \%$, and the completion rate was $>99 \%$. The quality and potential misclassification of the genotyping were assessed by regenotyping $10 \%$ of duplicate DNA samples that were randomly selected from the whole population and placed within the same reaction plates used for the study subjects. The concordance rate for the quality control samples was $100 \%$.

2.4. Statistical Analysis. We used SAS software (version 9.3; SAS Institute, Inc.) for the statistical analyses. $\chi^{2}$ statistics and the $t$-test were used to evaluate case-control differences in the distribution of risk factors. Variables were tested for normality with Shapiro-Wilk statistics. Skewed data, including age, BMI, TC, LDL-C, HDL-C, and tHcy, were log transformed and expressed as medians and interquartile ranges. A two tailed $P$ value of 0.05 was considered statistically significant. The odds ratios (ORs) and 95\% confidence intervals (CIs) for the associations between the SNPs and T2DM risk were estimated by unconditional logistic regression. HardyWeinberg equilibrium for genotypic distribution and linkage disequilibrium between loci were assessed by HaploView version 4.0 (Daly Lab at the Broad Institute, Cambridge, MA, USA) [17]. Associations between haplotypes ( $>1 \%$ frequency) and the risk of T2DM were evaluated by computing OR and 95\% CI using HAPSTAT, assuming an additive model, using the most common haplotype as the referent category [18]. Both univariate ANOVA and multivariate ANCOVA analyses adjusting for age, sex, BMI, and hypertension were performed to determine the effects of the MTHFR polymorphisms on serum tHcy levels in healthy controls. $P$ values (from the additive model or dominant model) of less than 0.0056 were deemed to be statistically significant after Bonferroni corrections.

\section{Results}

Characteristics of the study subjects are shown in Table 1. Cases and controls were evenly matched by age and gender. 
Cases were more probably to have higher BMI $\left(24.6 \mathrm{~kg} / \mathrm{m}^{2}\right.$ versus $23.1 \mathrm{~kg} / \mathrm{m}^{2}$ ) and hypertension (43.9\% versus $24.5 \%$ ). Besides, cases have significant lower levels of serum HDL-C and higher levels of serum total cholesterol and LDL-C than that in controls.

The associations of MTHFR variants and risk of T2DM are presented in Table 2 . The genotype distributions of these nine polymorphisms showed no deviation from the expected Hardy-Weinberg equilibrium among controls $(P>0.05)$. Of these SNPs, C allele of rs 9651118 conferred significant lowered risk of T2DM (crude OR $=0.71,95 \% \mathrm{CI}$ : $0.57-0.88, P=$ 0.002 ) compared with $\mathrm{T}$ allele, and rs1801131 AC genotype conferred significant increased risk of T2DM compared with AA genotype (crude OR $=1.29,95 \% \mathrm{CI}$ : $1.02-1.64, P=0.03$ ). These associations remained significant after adjustment for other risk factors (age, sex, BMI, and hypertension). But only rs9651118 was still significant after Bonferroni correction in dominant model (adjusted OR $=0.69,95 \%$ CI: $0.55-0.87$, $P=0.002)$. None of the other SNPs examined was associated with the risk of T2DM.

Six SNPs in the MTHFR gene (rs12121543, rs13306553, rs9651118, rs1801133, rs2274976, and rs1801131) were in linkage disequilibrium with $\mathrm{D}^{\prime}$ ranging from 0.79 to 0.99 and $r^{2}$ ranging from 0.04 to 0.86 . Subjects carrying the MTHFR CTCCGA haplotype had a significant reduced risk of T2DM $(\mathrm{OR}=0.73,95 \% \mathrm{CI}: 0.60-0.88, P=0.001)$ compared with those carrying the CTTTGA haplotype (Table 3 ). This association remained significant after adjustment for other risk factors and after Bonferroni correction.

Finally, we investigated the associations between the MTHFR SNPs and serum tHcy levels in the control population. Carriers of the mutant alleles of rs1801133 were significantly associated with increased serum level of tHcy [C/T: $12.8(10.9-14.4) \mu \mathrm{mol} / \mathrm{L} ; \quad \mathrm{T} / \mathrm{T}: 11.9(9.5-16.6) \mu \mathrm{mol} / \mathrm{L}]$ compared with carriers of the CC genotype [11.3(9.313.7) $\mu \mathrm{mol} / \mathrm{L}]$. Rs9651118 T/C and C/C genotypes [12.1(10.114.1) $\mu \mathrm{mol} / \mathrm{L}$; $11.3(9.3-13.4) \mu \mathrm{mol} / \mathrm{L}]$ conferred significant decreased serum level of tHcy in controls compared with TT genotype $[12.5(9.8-14.6) \mu \mathrm{mol} / \mathrm{L}]$. These associations were also significant in multivariate ANCOVA analyses. But after Bonferroni correction only rs1801133 was significantly associated with serum level of tHcy. None of the other studied SNPs was associated with serum tHcy level (Table 4).

\section{Discussion}

In this molecular epidemiologic study, polymorphisms in the MTHFR gene were fully studied for their association with susceptibility to T2DM and serum tHcy levels. We demonstrated that one genetic mutation (rs9651118) in MTHFR gene was significantly associated with risk of T2DM, and rs1801133 was significantly associated with serum tHcy levels in healthy population.

MTHFR gene is located on chromosome 1p36.3 in humans. To date, over 40 point genetic mutations in MTHFR gene have been identified, of which A1298C (rs1801131) and C677T (rs1801133) showed the most clinical significance. The MTHFR rs1801133 is a missense mutation that results in approximately $70 \%$ and $35 \%$ reduction of normal MTHFR enzyme activity in TT and CT genotype carriers, respectively [11]. MTHFR activity has an adverse effect on serum tHcy level. MTHFR rs1801133 TT genotype was also associated with elevated tHcy levels, predominantly in individuals who have a low plasma folate level $(<15.4 \mathrm{nmol} / \mathrm{L})$, compared to the normal genotype [19]. In our study, although $\mathrm{T}$ allele of rs1801133 has nonsignificant raised risk of T2DM (OR = 1.22, 95\%CI: 0.97-1.55), we found that the mutant alleles were associated with significantly higher level of tHcy in healthy controls. Besides, we observed that the average tHcy level in TT genotype carriers was $13.2 \mu \mathrm{mol} / \mathrm{L}$ which was higher than that in CT genotype carriers $(13.0 \mu \mathrm{mol} / \mathrm{L})$. These results were in accordance with previous studies.

A1298C (rs1801131) is a common mutation in the MTHFR gene that results in the conversion of adenine to cytosine, which results in reduction of MTHFR enzyme activity [20]. Few studies have evaluated the relationship between the MTHFR A1298C polymorphism and susceptibility of diabetes, and the results remain inclusive. Previous study suggested that MTHFR A1298C polymorphism is a risk factor for T2DM in Egyptian patients, while other failed to find any association in Taiwanese or Moroccan [21-23]. A recent Meta-analysis found that MTHFR A1298C has a significant association with diabetes in Asian population under dominant model but not in Caucasians [24]. The association between A1298C and serum homocysteine level was also controversial. Kumar et al. found that subjects with MTHFR rs1801131 CC genotype had significant higher level of homocysteine $(16.3 \mu \mathrm{mol} / \mathrm{L})$ compared with AA genotype $(14.4 \mu \mathrm{mol} / \mathrm{L})$ in Indian population, but this association was not found in Scotch $[25,26]$. In our study, although we found AC genotype had relatively higher risk of T2DM, the significance no longer exists after Bonferroni correction. In addition, we did not find its relation with homocysteine level.

Rs9651118 is located in the intron region in MTHFR gene with unknown genetic function. It was once reported that C allele of rs9651118 was associated with reduced lung cancer risk in never smokers, and a nominally significant association with schizophrenia in the form of haplotypes (rs1801133, rs17421511, rs17037396, and rs9651118) in Japanese population [27, 28]. Rs9651118 TT genotype was reported to confer significant elevated tHcy level compared with CC genotype in a population-based CoLaus study [29]. In our research, rs9651118 was associated with risk of T2DM, and we also found that carriers of TT genotype had nonsignificant higher level of tHcy relative to CC genotype carriers, which was in accordance with the previous study.

\section{Conclusion}

In conclusion, the present study suggests that MTHFR rs9651118 was associated with T2DM risk and MTHFR rs1801133 may affect serum tHcy levels. 
TABLE 2: ORs and 95\% CIs for T2DM in relation to polymorphisms of MTHFR gene.

\begin{tabular}{|c|c|c|c|c|c|c|}
\hline SNP & Genotypes & Cases, $n(\%)$ & Controls, $n(\%)$ & OR $(95 \% \mathrm{CI})^{*}$ & OR $(95 \% \mathrm{CI})^{\dagger}$ & $P$ trend $^{\ddagger}$ \\
\hline \multirow{4}{*}{ rs12121543 } & $\mathrm{CC}$ & $402(67.7)$ & $462(67.9)$ & 1.00 & 1.00 & \multirow{4}{*}{0.79} \\
\hline & $\mathrm{CA}$ & $177(29.8)$ & $194(28.5)$ & $1.04(0.82-1.33)$ & $1.07(0.83-1.38)$ & \\
\hline & AA & $15(2.5)$ & $24(3.5)$ & $0.71(0.37-1.37)$ & $0.77(0.38-1.54)$ & \\
\hline & $\mathrm{CA}+\mathrm{AA}$ & $192(32.3)$ & $218(32.1)$ & $1.01(0.80-1.27)$ & $1.04(0.81-1.33)$ & \\
\hline \multirow{4}{*}{ rs13306561 } & $\mathrm{TT}$ & $474(79.8)$ & $543(79.8)$ & 1.00 & 1.00 & \multirow{4}{*}{0.98} \\
\hline & TC & $116(19.5)$ & $131(19.3)$ & $1.01(0.76-1.33)$ & $1.04(0.77-1.39)$ & \\
\hline & $\mathrm{CC}$ & $4(0.7)$ & $6(0.9)$ & $0.73(0.20-2.61)$ & $0.58(0.15-2.21)$ & \\
\hline & $\mathrm{TC}+\mathrm{CC}$ & $120(20.2)$ & $137(20.1)$ & $1.00(0.76-1.31)$ & $1.01(0.76-1.35)$ & \\
\hline \multirow{4}{*}{ rs13306553 } & $\mathrm{TT}$ & $480(80.8)$ & $547(80.4)$ & 1.00 & 1.00 & \multirow{4}{*}{0.82} \\
\hline & $\mathrm{TC}$ & $110(18.5)$ & $129(19.0)$ & $0.97(0.73-1.28)$ & $0.97(0.72-1.30)$ & \\
\hline & $\mathrm{CC}$ & $4(0.7)$ & $4(0.6)$ & $1.08(0.27-4.36)$ & $1.06(0.25-4.50)$ & \\
\hline & $\mathrm{TC}+\mathrm{CC}$ & $114(19.2)$ & $133(19.6)$ & $0.97(0.73-1.28)$ & $0.97(0.73-1.30)$ & \\
\hline \multirow{4}{*}{ rs9651118 } & $\mathrm{TT}$ & $303(51.0)$ & $288(42.4)$ & 1.00 & 1.00 & \multirow{4}{*}{0.009} \\
\hline & $\mathrm{TC}$ & $231(38.9)$ & $300(44.1)$ & $0.73(0.58-0.93)$ & $0.72(0.56-0.93)$ & \\
\hline & $\mathrm{CC}$ & $60(10.1)$ & $92(13.5)$ & $0.62(0.43-0.90)$ & $0.59(0.40-0.87)$ & \\
\hline & $\mathrm{TC}+\mathrm{CC}$ & $291(49.0)$ & $392(57.6)$ & $0.71(0.57-0.88)$ & $0.69(0.55-0.87)$ & \\
\hline \multirow{4}{*}{ rs1801133 } & $\mathrm{CC}$ & $234(39.5)$ & $298(43.8)$ & 1.00 & 1.00 & \multirow{4}{*}{0.14} \\
\hline & $\mathrm{CT}$ & $293(49.4)$ & $312(45.9)$ & $1.20(0.95-1.51)$ & $1.22(0.96-1.56)$ & \\
\hline & $\mathrm{TT}$ & $66(11.1)$ & $70(10.3)$ & $1.22(0.83-1.77)$ & $1.23(0.83-1.84)$ & \\
\hline & $\mathrm{CT}+\mathrm{TT}$ & $359(60.5)$ & $382(56.2)$ & $1.20(0.96-1.50)$ & $1.22(0.97-1.55)$ & \\
\hline \multirow{4}{*}{ rs2274976 } & GG & $484(81.5)$ & $554(81.5)$ & 1.00 & 1.00 & \multirow{4}{*}{0.99} \\
\hline & GA & $105(17.7)$ & $119(17.5)$ & $1.00(0.75-1.34)$ & $1.02(0.75-1.38)$ & \\
\hline & $\mathrm{AA}$ & $5(0.8)$ & $7(1.0)$ & $0.78(0.25-2.49)$ & $0.62(0.19-2.04)$ & \\
\hline & $\mathrm{GA}+\mathrm{AA}$ & $110(18.5)$ & $126(18.5)$ & $0.99(0.75-1.32)$ & $1.00(0.74-1.34)$ & \\
\hline \multirow{4}{*}{ rs4846048 } & AA & $490(82.5)$ & $560(82.3)$ & 1.00 & 1.00 & \multirow{4}{*}{0.95} \\
\hline & AG & $99(16.7)$ & $114(16.8)$ & $0.99(0.74-1.33)$ & $1.01(0.74-1.38)$ & \\
\hline & GG & $5(0.8)$ & $6(0.9)$ & $0.97(0.29-3.19)$ & $1.39(0.40-4.88)$ & \\
\hline & $\mathrm{AG}+\mathrm{GG}$ & $104(17.5)$ & $120(17.6)$ & $0.99(0.74-1.32)$ & $1.03(0.76-1.39)$ & \\
\hline \multirow{4}{*}{ rs1801131 } & $\mathrm{AA}$ & $370(62.3)$ & 455 (66.9) & 1.00 & 1.00 & \multirow{4}{*}{0.04} \\
\hline & $\mathrm{AC}$ & $208(35.0)$ & $197(29.0)$ & $1.29(1.02-1.64)$ & $1.32(1.03-1.70)$ & \\
\hline & $\mathrm{CC}$ & $16(2.7)$ & $28(4.1)$ & $0.69(0.37-1.30)$ & $0.74(0.38-1.44)$ & \\
\hline & $\mathrm{AC}+\mathrm{CC}$ & $224(37.7)$ & $225(33.1)$ & $1.22(0.97-1.54)$ & $1.25(0.98-1.59)$ & \\
\hline \multirow{4}{*}{ rs17037396 } & $\mathrm{CC}$ & $456(76.8)$ & $521(76.5)$ & 1.00 & 1.00 & \multirow{4}{*}{0.88} \\
\hline & $\mathrm{CT}$ & $126(21.2)$ & $147(21.6)$ & $0.98(0.75-1.28)$ & $1.03(0.78-1.37)$ & \\
\hline & $\mathrm{TT}$ & $12(2.0)$ & $13(1.9)$ & $1.07(0.48-2.36)$ & $0.93(0.41-2.11)$ & \\
\hline & $\mathrm{CT}+\mathrm{TT}$ & $138(23.2)$ & $160(23.5)$ & $0.98(0.76-1.28)$ & $1.02(0.78-1.34)$ & \\
\hline
\end{tabular}

${ }^{*}$ Adjusted for age. ${ }^{\dagger}$ Adjusted for age, sex, BMI, and hypertension.

${ }^{\ddagger}$ Test of trend for the number of copies of the variant allele $(0,1$, and 2$)$.

TABLE 3: Association of haplotypes in the MTHFR gene with risk of T2DM.

\begin{tabular}{lcccr}
\hline Haplotype $^{*}$ & Cases, $\%$ & Controls, $\%$ & OR $^{\dagger}(95 \%$ CI $)$ & OR (95\% CI $)^{\ddagger}$ \\
\hline CTTTGA & 34.9 & 32.2 & 1.00 & 1.00 \\
CTCCGA & 26.9 & 35.3 & $0.73(0.60-0.88)$ & $0.71(0.58-0.87)$ \\
ATTCGC & 17.3 & 12.5 & $1.22(0.95-1.56)$ & $1.20(0.92-1.55)$ \\
ACTCAC & 8.6 & 8.2 & $0.92(0.67-1.25)$ & $0.93(0.67-1.29)$ \\
CTTCGA & 6.7 & 8.8 & $0.79(0.57-1.10)$ & $0.80(0.56-1.13)$ \\
\hline
\end{tabular}

* In the order rs12121543, rs13306553, rs9651118, rs1801133, rs2274976, and rs1801131.

${ }^{\dagger}$ Adjusted for age. ${ }^{\ddagger}$ Adjusted for age, sex, BMI, and hypertension. 
TABLE 4: Association between MTHFR polymorphisms and serum tHcy levels in health controls.

\begin{tabular}{|c|c|c|c|c|c|}
\hline \multirow{2}{*}{ SNP } & \multirow{2}{*}{$\mathrm{M} / \mathrm{m}$} & \multicolumn{3}{|c|}{$\mathrm{tHcy}(\mu \mathrm{mol} / \mathrm{L})$} & \multirow{2}{*}{$P^{*}$} \\
\hline & & MM & $\mathrm{Mm}$ & $\mathrm{mm}$ & \\
\hline rs12121543 & $\mathrm{C} / \mathrm{A}$ & $12.5(10.1-14.6)$ & $11.8(9.7-13.5)$ & $10.4(8.5-14.1)$ & 0.21 \\
\hline rs13306561 & $\mathrm{T} / \mathrm{C}$ & $12.1(9.9-14.4)$ & $10.5(8.4-13.7)$ & $12.3(10.0-14.4)$ & 0.87 \\
\hline rs13306553 & $\mathrm{T} / \mathrm{C}$ & $12.1(10.0-14.4)$ & $12.4(10.0-14.4)$ & $9.1(8.1-11.8)$ & 0.45 \\
\hline rs9651118 & $\mathrm{T} / \mathrm{C}$ & $12.5(9.8-14.6)$ & $12.1(10.1-14.1)$ & $11.3(9.3-13.4)$ & 0.02 \\
\hline rs1801133 & $\mathrm{C} / \mathrm{T}$ & $11.3(9.3-13.7)$ & $12.8(10.9-14.4)$ & $11.9(9.5-16.6)$ & 0.0002 \\
\hline rs2274976 & G/A & $12.1(9.9-14.4)$ & $12.3(10.0-14.1)$ & $10.7(8.4-13.7)$ & 0.84 \\
\hline rs4846048 & $\mathrm{A} / \mathrm{G}$ & $12.4(10.0-14.4)$ & $11.4(9.5-13.3)$ & $10.7(8.6-20.1)$ & 0.28 \\
\hline rs1801131 & $\mathrm{A} / \mathrm{C}$ & $12.5(10.1-14.6)$ & $11.8(9.7-13.6)$ & $10.8(8.6-14.1)$ & 0.26 \\
\hline rs17037396 & $\mathrm{C} / \mathrm{T}$ & $12.1(9.9-14.2)$ & $12.1(10.0-14.6)$ & $13.7(12.5-15.2)$ & 0.67 \\
\hline
\end{tabular}

* Adjusted for age, sex, BMI, and hypertension.

$\mathrm{M}$ indicates major allele; $\mathrm{m}$ indicates minor allele.

\section{Conflict of Interests}

The authors declare that there is no conflict of interests regarding the publication of this paper.

\section{References}

[1] H. King, R. E. Aubert, and W. H. Herman, "Global burden of diabetes, 1995-2025: prevalence, numerical estimates, and projections," Diabetes Care, vol. 21, no. 9, pp. 1414-1431, 1998.

[2] S. Wild, G. Roglic, A. Green, R. Sicree, and H. King, "Global prevalence of diabetes: estimates for the year 2000 and projections for 2030," Diabetes Care, vol. 27, no. 5, pp. 1047-1053, 2004.

[3] X. R. Pan, W. Y. Yang, G. W. Li, and J. Liu, "Prevalence of diabetes and its risk factors in China, 1994," Diabetes Care, vol. 20, no. 11, pp. 1664-1669, 1997.

[4] W. Yang, J. Lu, J. Weng et al., "Prevalence of diabetes among men and women in China," The New England Journal of Medicine, vol. 362, no. 25, pp. 1090-1101, 2010.

[5] Y. Bi, T. Wang, M. Xu et al., "Advanced research on risk factors of type 2 diabetes," Diabetes/Metabolism Research and Reviews, vol. 28, supplement 2, pp. 32-39, 2012.

[6] S. Mao, W. Xiang, S. Huang, and A. Zhang, "Association between homocysteine status and the risk of nephropathy in type 2 diabetes mellitus," Clinica Chimica Acta, vol. 431, pp. 206210, 2014.

[7] E. K. Hoogeveen, P. J. Kostense, P. J. Beks et al., "Hyperhomocysteinemia is associated with an increased risk of cardiovascular disease, especially in non-insulin-dependent diabetes mellitus: a population-based study," Arteriosclerosis, Thrombosis, and Vascular Biology, vol. 18, no. 1, pp. 133-138, 1998.

[8] M. Qureshi, H. Khsandwala, I. U. Haq, and K. Prasad, "Elevated levels of plasma homocysteine in hypertensive patients with diabetes mellitus," Journal of Cardiovascular Pharmacology and Therapeutics, vol. 8, no. 4, pp. 261-266, 2003.

[9] T. Huang, J. Ren, and J. Huang, "Association of homocysteine with type 2 diabetes: a meta-analysis implementing Mendelian randomization approach," BMC Genomics, vol. 14, article 867, 2013.

[10] M. O. Ebesunun and E. O. Obajobi, "Elevated plasma homocysteine in type 2 diabetes mellitus: a risk factor for cardiovascular diseases," The Pan African medical journal, vol. 12, p. 48, 2012.
[11] P. Frosst, H. J. Blom, R. Milos et al., "A candidate genetic risk factor for vascular disease: a common mutation in methylenetetrahydrofolate reductase," Nature Genetics, vol. 10, no. 1, pp. 111113, 1995.

[12] K. Shahzad, A. Hai, A. Ahmed, N. Kizilbash, and J. Alruwaili, "A structured-based model for the decreased activity of Ala222Val and Glu429Ala Methylenetetrahydrofolate Reductase (MTHFR) mutants," Bioinformation, vol. 9, no. 18, pp. 929936, 2013.

[13] S. C. Larsson, E. Giovannucci, and A. Wolk, "Folate intake, MTHFR polymorphisms, and risk of esophageal, gastric, and pancreatic cancer: a meta-analysis," Gastroenterology, vol. 131, no. 4, pp. 1271-1283, 2006.

[14] P. Li and C. Qin, "Methylenetetrahydrofolate reductase (MTHFR) gene polymorphisms and susceptibility to ischemic stroke: a meta-analysis," Gene, vol. 535, no. 2, pp. 359-364, 2014.

[15] B. Yang, S. Fan, and X. Zhi, "Associations of MTHFR gene polymorphisms with hypertension and hypertension in pregnancy: a meta-analysis from 114 studies with 15411 cases and 21970 controls," PLoS ONE, vol. 9, no. 2, Article ID e87497, 2014.

[16] American Diabetes Association, "Diagnosis and classification of diabetes mellitus," Diabetes Care, vol. 33, supplement 1, pp. S62-S69, 2010.

[17] J. C. Barrett, B. Fry, J. Maller, and M. J. Daly, "Haploview: analysis and visualization of LD and haplotype maps," Bioinformatics, vol. 21, no. 2, pp. 263-265, 2005.

[18] D. Y. Lin, D. Zeug, and R. Millikan, "Maximum likelihood estimation of haplotype effects and haplotype-environment interactions in association studies," Genetic Epidemiology, vol. 29, no. 4, pp. 299-312, 2005.

[19] P. F. Jacques, A. G. Bostom, R. R. Williams et al., "Relation between folate status, a common mutation in methylenetetrahydrofolate reductase, and plasma homocysteine concentrations," Circulation, vol. 93, no. 1, pp. 7-9, 1996.

[20] G. Friedman, N. Goldschmidt, Y. Friedlander et al., "A common mutation A1298C in human methylenetetrahydrofolate reductase gene: association with plasma total homocysteine and folate concentrations," Journal of Nutrition, vol. 129, no. 9, pp. 16561661, 1999.

[21] N. R. AbdRaboh, S. Badr, and S. Ali, "Prevalence of methylenetetrahydrofolate reductase C677T and A1298C polymorphisms in Egyptian patients with type 2 diabetes mellitus," Egyptian Journal of Medical Human Genetics, vol. 14, no. 1, pp. 87-93, 2013. 
[22] Y. Chang, W. Fu, Y. Wu, C. Yeh, C. Huang, and M. Shiau, "Prevalence of methylenetetrahydrofolate reductase C677T and A1298C polymorphisms in Taiwanese patients with Type 2 diabetic mellitus," Clinical Biochemistry, vol. 44, no. 17-18, pp. 1370-1374, 2011.

[23] H. Benrahma, O. Abidi, L. Melouk et al., "Association of the C677T polymorphism in the human methylenetetrahydrofolate reductase (MTHFR) gene with the genetic predisposition for type 2 diabetes mellitus in a Moroccan population," Genetic Testing and Molecular Biomarkers, vol. 16, no. 5, pp. 383-387, 2012.

[24] Y. Yan, H. Liang, S. Yang et al., "Methylenetetrahydrofolate reductase A1298C polymorphism and diabetes risk: evidence from a meta-analysis," Renal Failure, vol. 15, pp. 1-5, 2014.

[25] J. Kumar, S. K. Das, P. Sharma, G. Karthikeyan, L. Ramakrishnan, and S. Sengupta, "Homocysteine levels are associated with MTHFR A1298C polymorphism in Indian population," Journal of Human Genetics, vol. 50, no. 12, pp. 655-663, 2005.

[26] S. Narayanan, J. McConnell, J. Little et al., "Associations between two common variants C677T and A1298C in the methylenetetrahydrofolate reductase gene and of measures of folate metabolism and DNA stability (strand breaks, misincorporated uracil, and DNA methylation status) in human lymphocytes in vivo," Cancer Epidemiology Biomarkers and Prevention, vol. 13, no. 9, pp. 1436-1443, 2004.

[27] M. D. Swartz, C. B. Peterson, P. J. Lupo et al., "Investigating multiple candidate genes and nutrients in the folate metabolism pathway to detect genetic and nutritional risk factors for lung cancer," PLoS ONE, vol. 8, no. 1, Article ID e53475, 2013.

[28] A. Yoshimi, B. Aleksic, Y. Kawamura et al., "Gene-wide association study between the methylenetetrahydrofolate reductase gene (MTHFR) and schizophrenia in the Japanese population, with an updated meta-analysis on currently available data," Schizophrenia Research, vol. 124, no. 1-3, pp. 216-222, 2010.

[29] F. Marti, P. Vollenweider, P. Marques-Vidal et al., "Hyperhomocysteinemia is independently associated with albuminuria in the population-based CoLaus study," BMC Public Health, vol. 11, article 733, 2011. 


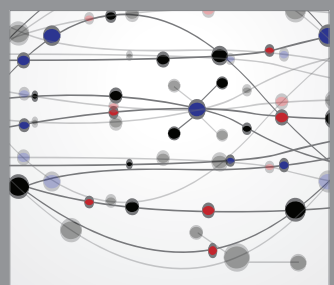

The Scientific World Journal
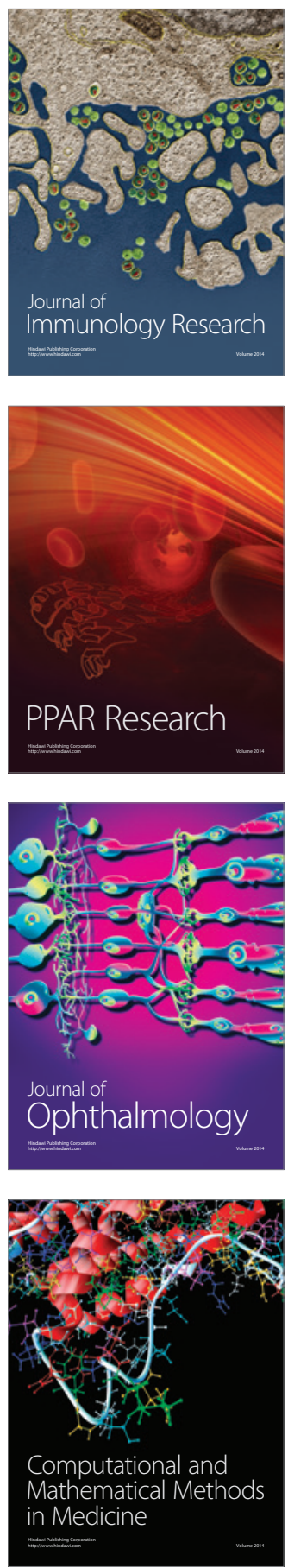

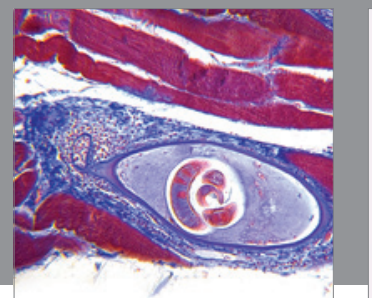

Gastroenterology

Research and Practice
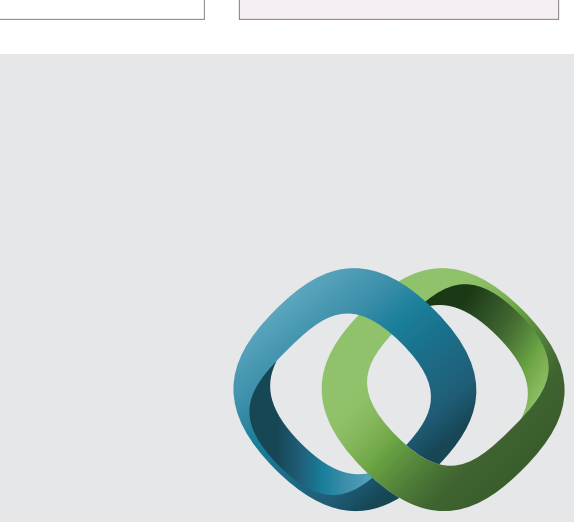

\section{Hindawi}

Submit your manuscripts at

http://www.hindawi.com
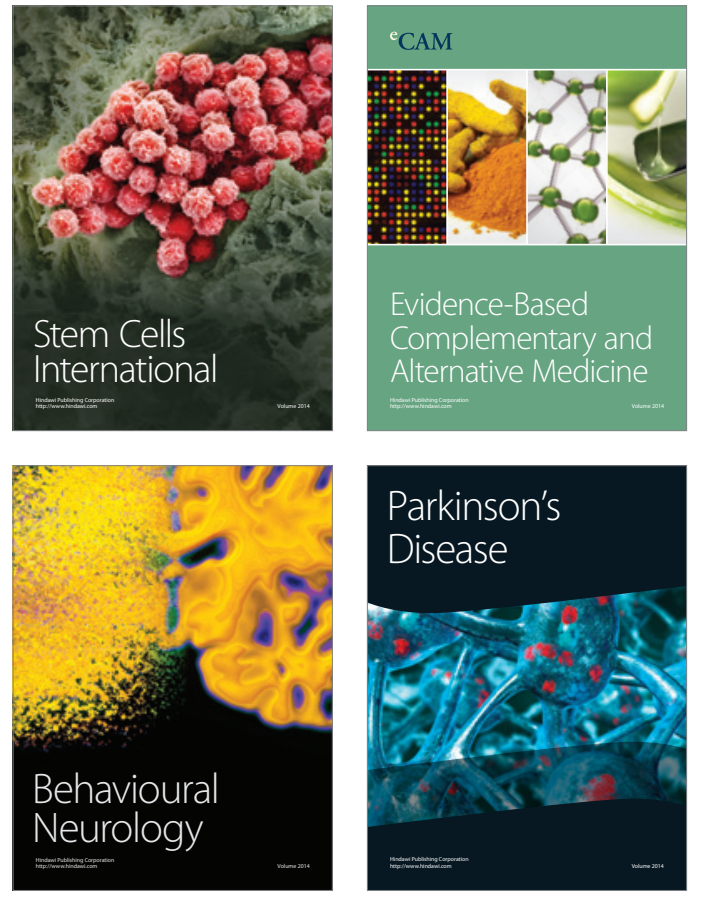
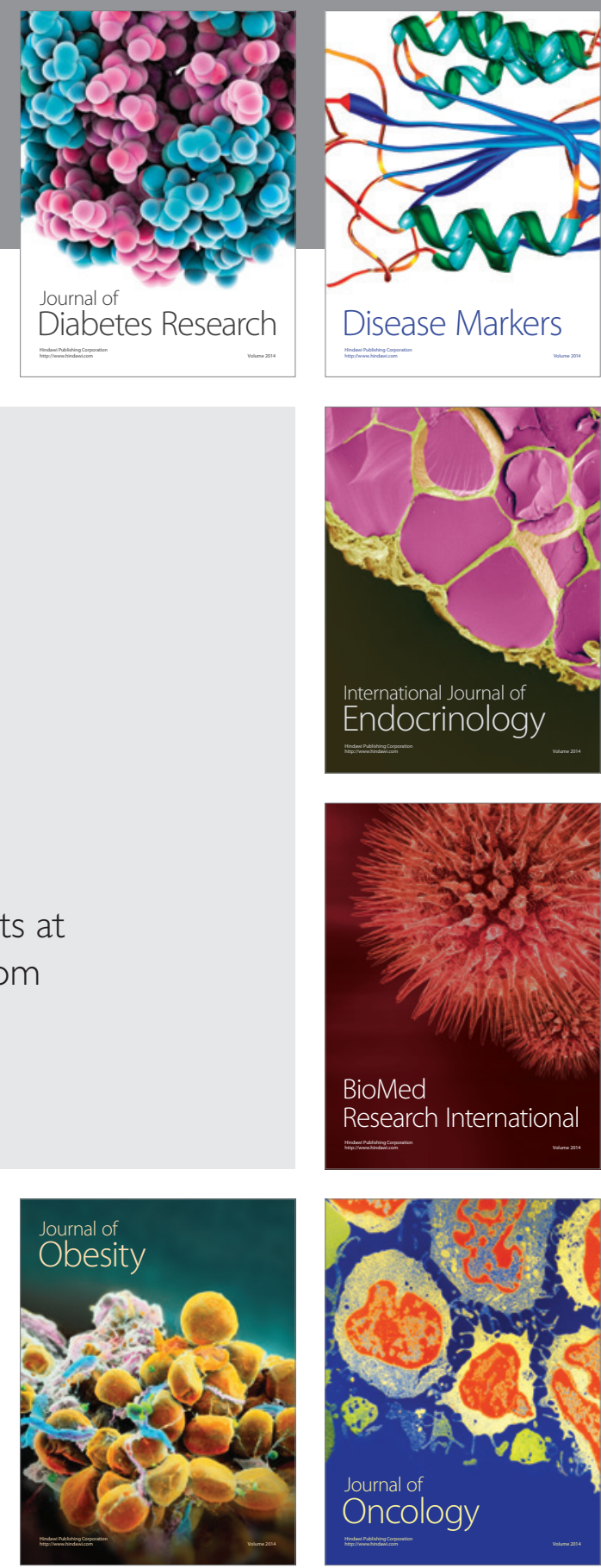

Disease Markers
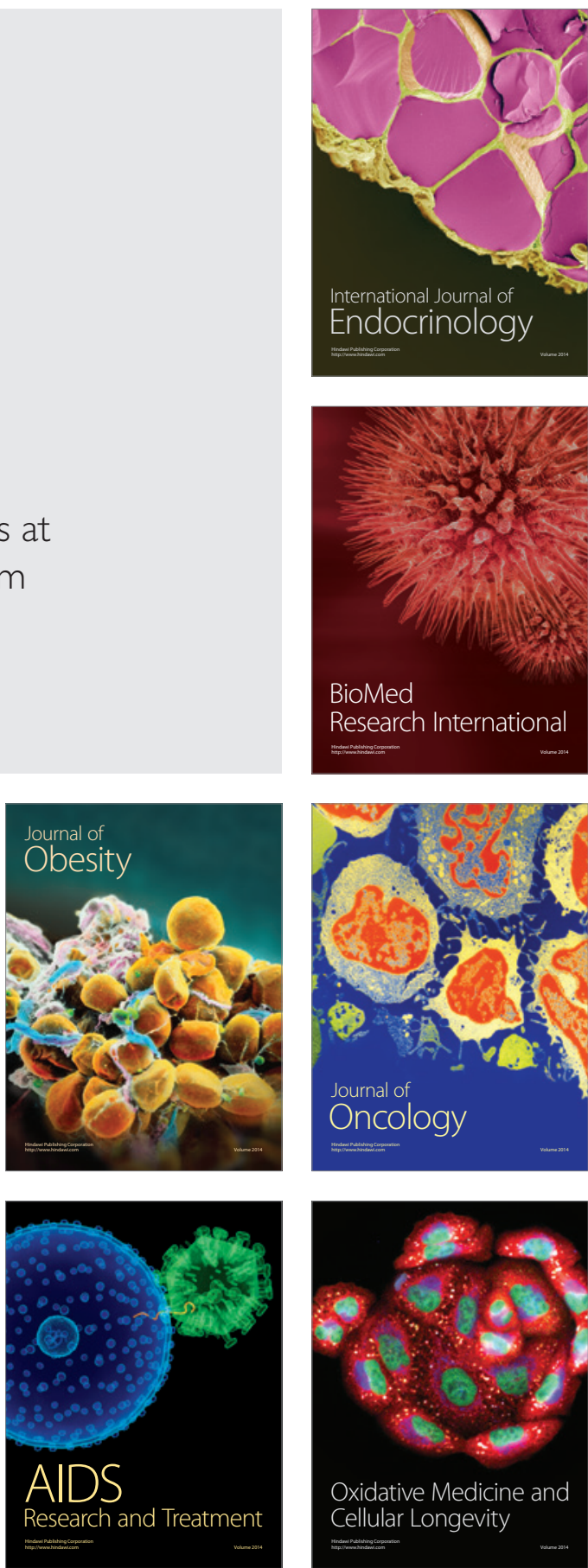